\title{
$\Omega$
}

Laura Petrarca ${ }^{1}$, Tiago Jacinto ${ }^{2,3,4}$, Raffaella Nenna ${ }^{1}$

廿]

'Dept of Paediatrics and Infantile Neuropsychiatry, "Sapienza" University of Rome, Rome, Italy.

2Dept of Allergy, Instituto and Hospital CUF, Porto, Portugal.

${ }^{3}$ CINTESIS - Center for Health Technology and Services Research, Faculty of Medicine, University of Porto, Porto, Portugal.

${ }^{4}$ Dept of Cardiovascular and Respiratory Sciences, Porto Health School, Porto, Portugal.

\section{The treatment of acute bronchiolitis: past, present and future}

\section{Landmark papers in respiratory medicine}

Lower respiratory tract infections are a common cause of hospitalisation in infants. It is estimated that infants younger than 12 months with bronchiolitis account for $18 \%$ of all paediatric admission [1], representing a great burden to industrialised healthcare systems each winter.

Bronchiolitis is defined as the first respiratory tract infection in infants younger than 12 months. Clinically, it can be manifested by cough, tachypnoea, apnoea, increased respiratory effort, fever, nasal congestion and rhinorrhoea. On chest auscultation, the key feature is diffuse bilateral inspiratory crackles. The most common virus detected in children with bronchiolitis is respiratory syncytial virus (RSV).

\section{The past}

Bronchiolitis was initially called "capillary bronchitis" and was not well accepted as a defined pathological entity because both pathologists and paediatricians believed that a lesion limited to the finest bronchi could never exist alone, and the inflammatory process would have involved the alveoli, resulting in a picture of bronchopneumonia.

In 1941, Hubble and Osborn [2] described the clinical picture of acute bronchiolitis in the British Medical Journal. They identified the disease as concomitant to influenza spread in children younger than 2 years, most often among poorer classes, probably due to malnutrition, confined living space and closer contact with infections.

The treatment options available between the end of the 19th century and the early 20th, with the exception oxygen, would today be considered strange and rather fanciful. Those options would have also, among the others, fresh air, hydrotherapy, steam tents, alcohol, digitalis, emetics, stimulants, mustard poultices, venesection, blood transfusion, sedatives and cough mixtures. However, already in the Hubble and Osborn [2] paper (back in 1940), the acute bronchiolitis treatment was mainly supportive, including feeding children in warm, moist setting and ensuring an adequate oxygen supply. Alcohol was reported to be the best sedative: brandy, whisky or port at a dose of 30 minims $(\sim 1.85 \mathrm{~mL})$ to 1 drachm $(\sim 3.7 \mathrm{~mL})$ twice
Cite as: Petrarca $L$, Jacinto $T$ Nenna R. The treatment of acute bronchiolitis: past, present and future. Breathe 2017; 13: e24-e26. 
or three times per day in 1-year-old children. No other sedatives were recommended as depression in respiratory excursion could lead to death: "any derivate of opium may bring a sleep which is final". In the treatment of acute bronchiolitis, the authors considered four different factors: the infection, the obstructive dyspnoea, the cyanosis and the collapse. For the infection control, they suggested using sulfapyridine in full dosage for at least a week. The management of obstructive dyspnoea included steam; adrenalin was recommended to control bronchial spasm while atropine was recommended to decrease bronchiolar exudate. In addition, bronchoscopic aspiration, performed by experts in paediatric patients, was encouraged to prevent "self-drownage". Cyanosis treatment included oxygen supplementation, delivered through a Woulfe's bottle of warm water and nasal catheter. All these therapies aimed at preventing the collapse of the cardiorespiratory system. In the worst case of such a collapse, the best approach seemed to be the stimulation of the circulatory system with Coramine (nikethamide) and the reduction of preloading with venesection.

\section{The present}

Since 1950, even though several efforts have been made to achieve an effective treatment for bronchiolitis, it has remained mainly supportive [3].

The recent practice guidelines of the American Academy of Pediatrics (AAP) [4] and the UK National Institute for Health and Care Excellence (NICE) guidelines [5] recommend the administration of either nasogastric or intravenous fluid in infants with fluid intake reduction and oxygen supplementation when the oxygen saturation is either $<90 \%$ (AAP reference value) or $<92 \%$ (NICE guidelines reference value). AAP and NICE both agree on avoiding the use of nebulised albuterol, salbutamol and adrenaline, and the administration of systemic corticosteroids and antibiotics.

Regarding the role of hypertonic saline, the AAP guidelines do not recommend its use in the emergency department but only in children hospitalised. However, the NICE guidelines discourage its use. What emerges from a recent meta-analysis is that 3\% hypertonic saline seems to slightly reduce the length of hospital stay [6]. However, the authors reported the difficulty in analysing the results from different studies in which the definition of bronchiolitis differs between countries, together with the level of standard care.

By analysing the most recent meta-analysis, the latest Cochrane review of the use of bronchodilators for the treatment of bronchiolitis showed no effect on oxygen saturation, and no reduction in hospital admission or in length of hospitalisation [7]. In addition, antibiotics seem not to have an effect on the course of the disease [8].
Neither systemic nor inhaled corticosteroids seem to have a role in preventing hospital admission or in reducing hospital stay in infants with bronchiolitis. However, the latest Cochrane review suggested that combining a high dose of systemic dexamethasone and adrenaline could reduce outpatient admissions from moderate bronchiolitis. This result has been shown with a single large trial that needs further and larger investigation [9].

The use of surfactant in critically ill infants appears not to reduce the duration of mechanical ventilation when compared with placebo or treatment absence although the duration of intensive care unit stay was shorter in the surfactant group and there was a better effect of surfactant on gas exchange [10].

The most important innovation in bronchiolitis treatment is noninvasive oxygen support with a high-flow nasal cannula (HFNC), which, so far, has shown to be safe, feasible [11] and costeffective [12]. HFNC can reduce airway resistance by delivering humidified and heated oxygen at a higher inspired gas flow while also providing some level of continuous positive airway pressure to improve ventilation. This may reduce the need for invasive respiratory support, thus potentially lowering costs, with clinical advantages and fewer adverse effects. However, in order to prove HFNC effectiveness, further evidence should be provided.

\section{The future}

A more effective opportunity to prevent bronchiolitis due to RSV relies on a RSV vaccine. In the 1960s, the first formalin-inactivated RSV vaccine proved to be not only ineffective but also self-defeating, causing a vaccine-enhanced disease [13].

Several vaccines candidates are now under evaluation in pre-clinical or clinical trials, including protein subunits, viral vectors and live attenuated viruses [14]. It is also under discussion who is going to be vaccinated, pregnant mothers or the paediatric population. How effective these vaccines could be in preventing RSV-related disease and complications is currently not predictable, but certainly, increased knowledge of RSV immunopathogenesis could lead to the development of the most appropriate vaccine.

A possible upcoming treatment for RSV bronchiolitis could be the antiviral therapy. The targets of molecules for the treatment of RSV include fusion inhibitors, RSV polymerase, the $\mathrm{N}$-terminal region of the nucleocapsid protein and influenza polymerase, but they are still in the early experimental stages [15]

Ideally, a possible future strategy for the treatment of bronchiolitis may also include the use of a nebulised mucolytic drug that should be able to dissolve the debris that occludes the lumen of the terminal and respiratory bronchioles. Many studies 
have been reported on the use of DNAse but their results are still debated $[16,17]$.

\section{Conclusion}

Bronchiolitis remains a frequent cause of hospital admission in children, often causing severe disease even in previously healthy infants, thus implying a great burden for healthcare systems. Albeit that the treatment for bronchiolitis has been investigated for a long time, a firm conclusion on the best treatment has not been reached yet. The most promising treatment available nowadays for bronchiolitis is HFNC, which has proved to be safe, feasible and cost-effective [11, 12]. A desirable solution for the near future is to prevent children from being affected, perhaps with an effective vaccine.

\section{References}

1. Hasegawa K, Tsugawa Y, Brown DF, et al. Trends in bronchiolitis hospitalizations in the United States, 2000-2009. Pediatrics 2013; 132: 28-36.

2. Hubble D, Osborn GR. Acute bronchiolitis in children. BrMed J 1941; 1: 107-126.1.

3. Castro-Rodriguez JA, Rodriguez-Martinez CE, Sossa-Briceño MP. Principal findings of systematic reviews for the management of acute bronchiolitis in children. Paediatr Respir Rev 2015; 16: 267-275.

4. Ralston SL, Lieberthal AS, Meissner HC, et al. Clinical practice guideline: the diagnosis, management, and prevention of bronchiolitis. Pediatrics 2014; 134: e1474-e1502.

5. National Institute for Health and Care Excellence. Bronchiolitis in children. NG9. London, National Institute for Health and Clinical Excellence, 2015.

6. Maguire C, Cantrill H, Hind D, et al. Hypertonic saline (HS) for acute bronchiolitis: systematic review and meta-analysis. BMC Pulm Med 2015; 15: 148

7. Gadomski AM, Scribani MB. Bronchodilators for bronchiolitis. Cochrane Database Syst Rev 2014; CD001266.

8. Farley R, Spurling GKP, Eriksson L, et al. Antibiotics for bronchiolitis in children under two years of age. Cochrane Database Syst Rev 2014; CD005189.

9. Fernandes RM, Bialy LM, Vandermeer B, et al. Glucocorticoids for acute viral bronchiolitis in infants and young children. Cochrane Database Syst Rev 2013; CD004878
10. Jat KR, Chawla D. Surfactant therapy for bronchiolitis in critically ill infants. Cochrane Database Syst Rev 2015; CD009194.

11. Beggs S, Wong ZH, Kaul S, et al. High-flow nasal cannula therapy for infants with bronchiolitis. Cochrane Database Syst Rev 2014; CD009609.

12. Heikkilä P, Forma L, Korppi M. High-flow oxygen therapy is more cost-effective for bronchiolitis than standard treatment-A decision-tree analysis. Pediatr Pulmonol 2016; 51: 1393-1402.

13. Graham BS. Vaccines against respiratory syncytial virus: the time has finally come. Vaccine 2016; 34 : 3535-3541.

14. Chiu C. Novel immunological insights in accelerating RSV vaccine development. Vaccine 2017; 35: 459-460.

15. Simões EA, DeVincenzo JP, Boeckh $M$, et al. Challenges and opportunities in developing respiratory syncytial virus therapeutics. J Infect Dis 2015; 211: Suppl. 1, S1-S20.

16. Enriquez A, Chu IW, Mellis C, et al. Nebulised deoxyribonuclease for viral bronchiolitis in children younger than 24 months. Cochrane Database Syst Rev 2012; 11: CD008395.

17. Nenna R, Tromba V, Berardi R, et al. Recombinant deoxyribonuclease treatment in hospital management of infants with moderate-severe bronchiolitis. Eur J Inflammation 2009; 7: 169-174. 